\title{
IMPACTO ANTRÓPICO EN LA CALIDAD DEL AGUA SUPERFICIAL DE LA CUENCA MEDIA DEL ARROYO CLAROMECÓ, ARGENTINA
}

\author{
M.E. CARBONE ${ }^{*}$, B. GARCÍA-MARTÍNEZ², \\ J. MARCOVECHIO' ${ }^{1}$, M.C. PICCOLO' ${ }^{1}$, G.M.E. PERILLO ${ }^{1}$ \\ ${ }^{1}$ Instituto Argentino de Oceanografía, Camino La Carrindanga km 7, \\ 8000 Bahía Blanca, Argentina. \\ ${ }^{2}$ Facultad de Geografía e Historia, Universidad de Sevilla, España.
}

RESUMEN. Los cursos fluviales de la cuenca media del Arroyo Claromecó en su recorrido atraviesan la localidad de Tres Arroyos con más de 59000 habitantes, donde se localiza una planta industrial y una planta de tratamiento de efluentes urbanos. El objetivo de este trabajo es analizar la calidad de agua de los cursos superficiales de la cuenca media para estudiar el impacto humano en este sector del sistema fluvial. Se seleccionaron 5 estaciones de muestreo para la concentración de metales pesados en el sedimento de los cauces y en cuatro de ellas se obtuvieron valores de nutrientes en agua. Estas mediciones se realizaron durante agosto del 2007 y se repitieron durante agosto del 2009. La determinación de nutrientes se realizó mediante el uso de un autoanalizador Technicon AutoAnalyzer II secuencial y por espectrofotometría se analizó la Materia Orgánica Particulada. Los valores de nutrientes hallados presentaron mayor variabilidad entre los años 2007 y 2009. Los máximos se observaron durante el primer año de medición, mientras que el máximo de amonio se observó en el colector principal para el año 2009. Se detectaron variables superiores a los niveles guía de protección de la vida acuática, como el caso de nitratos y fosfatos en el Arroyo Claromecó, tendencia que se mantuvo en casi todos los parámetros químicos, lo que se correspondería con descargas cloacales y secundariamente industriales de las plantas procesadoras por las que atraviesa este curso fluvial en el área urbana y periurbana de la localidad de Tres Arroyos. Los sedimentos fluviales analizados presentaron valores de metales pesados bajos-intermedios. El análisis de las variables físicas y químicas del agua reflejan para los afluentes del Arroyo Claromecó las características de sustrato y la influencia del uso agrícola ganadero circundante. El curso principal presenta un predominio del uso urbano e industrial a partir del mayor aporte de nutrientes a sus aguas, provenientes de descargas domésticas directas sin tratamiento. 


\section{Human impact on the quality of the surface waters of the Claromecó Creek mid-basin, Argentina}

ABSTRACT. The creeks of the middle basin of the Arroyo Claromecó cross the town of Tres Arroyos with over 59000 inhabitants, where an industrial plant and a wastewater treatment plant city is located. The aim of this paper is to analyze the quality of surface water courses of the basin to study human impact on this sector of the river system. We selected 5 sampling stations for the concentration of heavy metals in the sediment of rivers. In four of them values of nutrients in the water were obtained. These measurements were conducted during August 2007 and repeated in August 2009. The determination of nutrients was performed using a sequential Technicon Autoanalyzer II and the Particulate OrganicMatter was analyzed by spectrophotometry. The nutrient values showed greater variability between 2007 and 2009. The maxima were observed during the first year of measurement, while the maximum of ammonium was observed in the main sewer in 2009. Variables were detected above guideline levels for the protection of aquatic life, as the case of nitrates and phosphates in the Arroyo Claromecó. This trend was maintained for almost all the chemical parameters, which would correspond to secondary sewage and industrial discharges from plants processing by the river that runs through this course in peri-urban areas of the town of Tres Arroyos. The analyzed river sediments showed low to intermediate values of heavy metals. For the tributaries of the Arroyo Claromecó, the analysis of the physical and chemical variables of water reflects substrate characteristics and the influence of surrounding agricultural livestock. The main course presents a predominance of urban and industrial use from the increased supply of nutrients to the water, that come directly from untreated domestic discharges.

Palabras claves: calidad de agua, cursos fluviales, Arroyo Claromecó, usos del suelo. Key words: water quality, fluvial courses, Claromecó Creek, land use.

* Correspondencia: Instituto Argentino de Oceanografía, Camino La Carrindanga km 7, 8000 Bahía Blanca, Argentina. E-mail: ecarbone@criba.edu.ar

\section{Introducción}

El incremento de población y sus consecuentes necesidades materiales de desarrollo imponen progresivamente mayores exigencias a los sistemas hídricos, ya que la intensificación del manejo del agua en una cuenca se traduce en trastornos ambientales que modifican los sistemas acuáticos, manifestándose sobre la productividad de sistemas naturales y antropizados. Esta preocupación, existente desde hace algunas décadas, ha 
potenciado el estudio de los sistemas hídricos desde una perspectiva integral del manejo de cuencas hidrográficas, dando una especial significación a la dimensión ambiental, en particular a los aspectos vinculados a la calidad del agua de los cursos fluviales.

Las causas del deterioro de la calidad de las aguas en cuencas hídricas, estudiado a nivel mundial desde hace varios años, incluyen entre otras la descarga a los cursos de aguas superficiales de líquidos cloacales y pluviales, el vertido de efluentes industriales con nulo o escaso tratamiento, el aporte del arrastre de suelo con contenido de plaguicidas, fertilizantes, vertido de desechos orgánicos pecuarios y otras sustancias. Como consecuencia, las aguas experimentan cambios físicos y químicos que afectan a las distintas comunidades que en ellas viven (Perona et al., 1999; Márquez y Martínez, 2000; Mencio y Manplat, 2008; Panno et al., 2008). En la contaminación de las aguas tienen una gran incidencia las actividades domésticas, agrícolas e industriales desarrolladas por el ser humano. Las actividades domésticas contribuyen mediante el aporte de restos orgánicos, tierra procedente del lavado de vegetales, detergentes, grasas, sales o jabones (Herzer et al., 2003). También los residuos fecales humanos que, además de microorganismos patógenos, contienen fosfatos y cloruros que contribuyen en gran medida a la pérdida de la calidad del agua (Vega et al., 1998; Mason y Miller, 2004).

El estudio de la calidad del agua tanto superficial como subterránea aporta información con respecto a sus posibles usos (consumo humano, animal, riego, recreación, etc.). En la actualidad, la diversidad en calidad y cantidad de los desechos sólidos y efluentes vertidos en ambientes acuáticos es tal que exige un análisis de múltiples variables que interactúan entre sí (Mancini et al., 2003). De este modo, la calidad de los recursos hídricos por diversos usos se ve afectada en diferente grado en muchas cuencas de Argentina (Lerda y Prosperi, 1994; Herzer et al., 2003). Los usos agrícolas, industriales y urbanos son responsables de una gran parte de los contaminantes vertidos a las aguas de los sistemas hídricos. Entre ellos destaca el aporte de grandes cantidades de nitratos y nitritos. Según Goic y Rojas (2004), estos nitratos y nitritos provienen en su mayoría de las explotaciones agrícolas circundantes a los cursos fluviales. De esta forma, la calidad del agua de los espacios naturales se ve alterada y los impactos sobre ella deben ser evaluados.

La calidad del agua de la cuenca del Arroyo Claromecó comenzó a analizarse en el año 2005 (Carbone et al., 2005). A partir de los datos obtenidos, se determinó que el 14.3\% de las aguas eran buenas para el riego, mientras que el $85.7 \%$ resultaban aceptables. La interpretación de los análisis de agua para riego permitió determinar que las aguas de la cuenca alta y media presentaban las mejores condiciones para los cereales. Las aguas del Primer Brazo no eran recomendables por su alta salinidad y sodicidad. Para reducir al mínimo el posible impacto negativo del sodio en el suelo es indispensable implementar, previo al riego, prácticas de manejo conservacionista de suelos y mejorar así la condición superficial de los mismos. Los análisis realizados indicaron la existencia de aguas bicarbonatadas sódicas en todos los casos. En cuanto a su calidad para riego, no tenían efectos tóxicos para los cultivos de maíz y soja (INTA, 1995). En este trabajo de investigación se evalúa la calidad de agua de los cursos superficiales y sedimentos que integran la red hídrica del arroyo Claromecó en su cuenca media, con el objeto de 


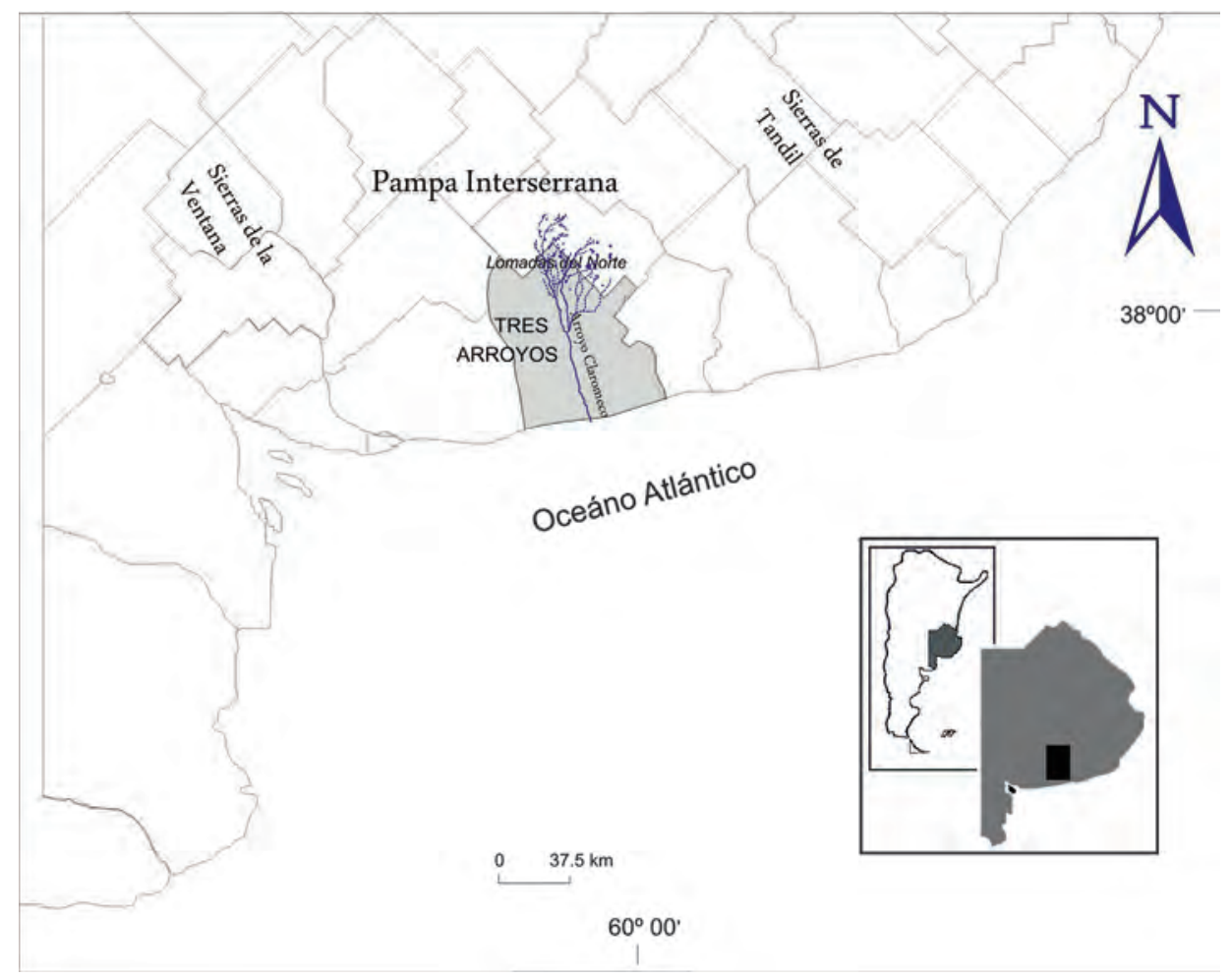

Figura 1. Localización del Arroyo Claromecó en el sudeste de la provincia de Buenos Aires.

determinar si existe impacto antrópico en la misma y cuál es el estado actual de la cuenca en relación a los límites tolerables para la protección de la vida acuática.

\section{2. Área de estudio}

La cuenca del Arroyo Claromecó se encuentra localizada en el sudeste de la provincia de Buenos Aires. El río nace en la denominada Pampa de Juárez o llanura interpuesta, zona de escasa pendiente ubicada entre dos sistemas serranos, el de Ventania y el de Tandilia (Fig. 1). Cabe destacar que, aunque el arroyo Claromecó no es uno de los cursos principales de la provincia, se encuentra en una región agrícola-ganadera muy importante de Argentina: la llanura pampeana.

El área de la cuenca del Arroyo Claromecó $\left(3054 \mathrm{~km}^{2}\right)$ participa con el 8\% en la producción de grano nacional ya que posee algunas de las tierras trigueras más fértiles y de mayor rendimiento del país (Carbone, 2004). El Arroyo Claromecó se conforma a partir de la confluencia de tres cursos de agua: por el oeste el Tercer Brazo $(77 \mathrm{~km})$, al centro el Segundo Brazo (61 km) y por el este el Primer Brazo $(24 \mathrm{~km})$. Los cursos de agua de los tres brazos y el arroyo Claromecó, con una longitud de $59 \mathrm{~km}$ y un caudal 
de $0.63 \mathrm{~m}^{3} \mathrm{~s}^{-1}$, tienen régimen fluvial permanente (Carbone et al., 2006, García Martínez et al., 2008) y en su recorrido atraviesan la localidad de Tres Arroyos con 59000 habitantes (INDEC, 2010). Estos cuerpos de agua a su vez encuentran en su paso un parque industrial, una planta depuradora de efluentes cloacales y una fábrica de productos cárnicos, que desagua sus desechos directamente a los cursos mencionados.

El área de la cuenca media del Arroyo Claromecó se ha caracterizado tradicionalmente por el predominio de un sistema mixto agrícola-ganadero. Sin embargo, y como ocurrió a lo largo de toda la región pampeana, en los últimos años se ha producido una tendencia a prolongar los ciclos agrícolas con respecto al período de uso ganadero; pero también, se han incorporado nuevas superficies con baja aptitud para la actividad agrícola (Forján, 2004; Portela et al., 2006). Este uso más intensivo conlleva una aplicación cada vez más recurrente de fertilizantes nitrogenados y herbicidas y cabe destacar que estos últimos se aplican a través de fumigaciones aéreas, cuando las condiciones de suelo son de humedad severa.

La fertilización con nitrógeno en el sur de la provincia de Buenos Aires ha presentado una importante variabilidad de respuesta según la campaña y las localidades evaluadas. Tanto en la campaña 2005/06 como en la 2008/09 se observó una disminución de aplicación mientras que en la campaña 2006/07 como en la 2007/08 fueron positivas y variables de acuerdo al sitio (Zamora et al., 2009; Andrade et al., 2005). El uso agrícola promedio de los suelos, que históricamente representaba un $50 \%$ y a principios de la década de los años 80 se encontraba cercano al 60\%, ha llegado en la actualidad a ocupar el 78\% de la superficie total de los establecimientos promedio de la región con valores máximos en Tres Arroyos (CEI Barrow, 2003).

\section{Metodología}

Los sitios de muestreo en la cuenca media del Arroyo Claromecó se establecieron en los tres brazos y en el arroyo en el que confluyen (Fig. 2). En cada uno de los sitios se obtuvieron muestras de agua y sedimento. Este procedimiento se realizó durante agosto del 2007 y se repitió durante agosto del 2009. Las muestras se analizaron de forma completa en el laboratorio de Química Marina del Instituto Argentino de Oceanografía.

Para determinar las concentraciones de nutrientes, clorofila "a", feopigmentos y materia orgánica particulada (MOP) en agua se siguieron los siguientes métodos: nitritos (Grasshof et al., 1983), fosfatos (Eberlein y Kattner, 1987), silicatos (Technicon, 1987), amonio (Richard y Kletsch, 1964) y MOP (oxidación húmeda y espectrofotometría según Strickland y Parsons, 1968). La determinación de nutrientes se realizó mediante el uso de un autoanalizador Technicon AutoAnalyzer II secuencial. Para analizar los pigmentos se siguió el método ya mencionado donde se extraen los pigmentos con solvente orgánico. La lectura de absorbancia de los extractos se llevo a cabo con un espectrofotómetro de UV-VIS, Beckman DU-2. La determinación de MOP se realizó por oxidación húmeda con una mezcla de ácido sulfúrico concentrado y dicromato de potasio, adaptada para espectrofotometría. Asimismo, en cada una de las estaciones se midió en el agua los siguientes parámetros físicos y químicos: temperatura, oxígeno 


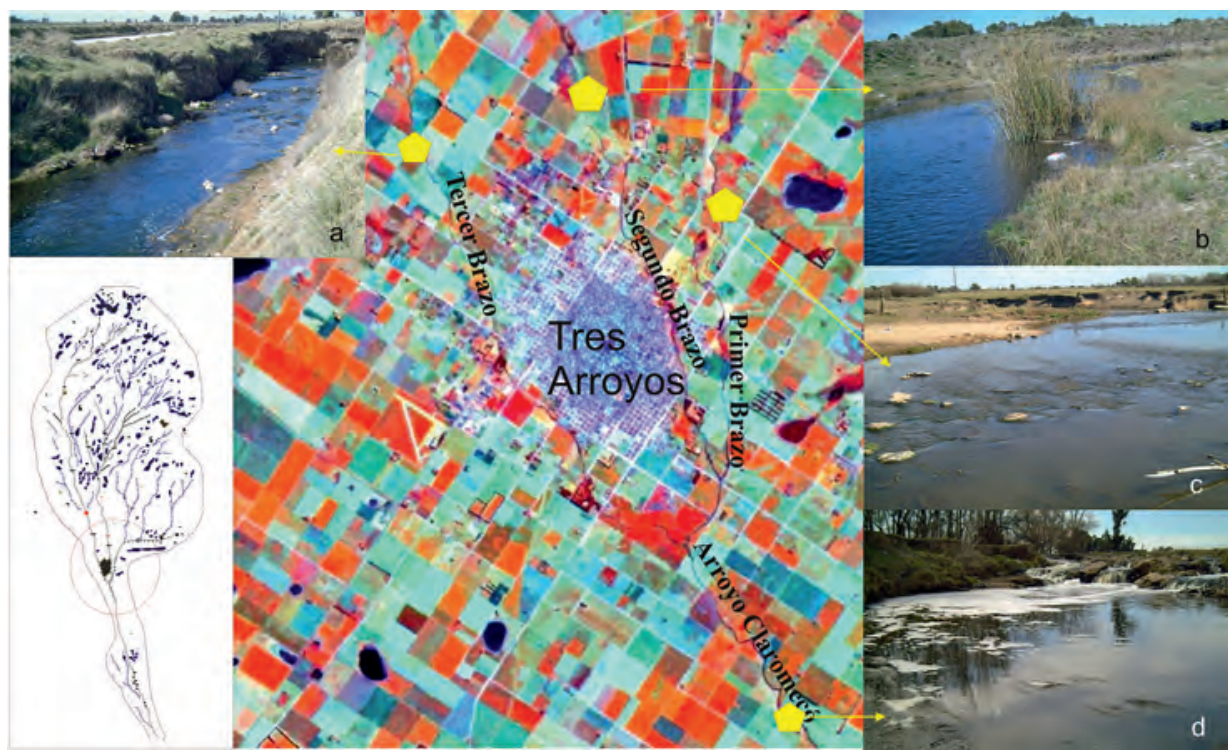

Figura 2. Ubicación de los sitios de muestreos en la cuenca media del Arroyo Claromecó. Las fotografías de cada colector corresponden al 26 de agosto del 2009.

disuelto, $\mathrm{pH}$, conductividad, turbidez y salinidad utilizando una sonda multisensor Horiba U-10. Los metales $\mathrm{Cd}, \mathrm{Pb}, \mathrm{Cr}, \mathrm{Ni}, \mathrm{Zn}, \mathrm{Mn}$ y Fe en el sedimento de los cauces fueron determinados usando el método propuesto por Dalziel y Baker (1983) modificado por Marcovecchio et al. (1988), reemplazando por ácido perclórico el peróxido de hidrogeno, empleado originalmente para mejorar la oxidación de la muestras, para lo cual se utilizó un Espectrofotómetro de Absorción Atómica (EAA) Perkin Elmer Modelo 2380.

\section{Resultados}

En la tabla 1 se presentan los valores mínimos y máximos de las variables físicas y químicas obtenidos durante los muestreos. La temperatura del agua de los arroyos fue mayor en agosto de 2009 donde los valores registraron casi $5^{\circ} \mathrm{C}$ más que en 2007 . Los valores oscilan en este muestreo entre 14 y $15.8^{\circ} \mathrm{C}$ para el Tercer Brazo y el Arroyo Claromecó respectivamente, mientras que para 2007 los registros no superan los $10.1^{\circ} \mathrm{C}$ (Fig. 3a). Los valores de $\mathrm{pH}$ para ambas mediciones se mantuvieron dentro de los niveles guía para la protección de la vida acuática de acuerdo a la Ley Nacional № 24054 de la República Argentina.

En cuanto a la concentración de oxígeno disuelto en el agua de los ríos, ésta va a depender principalmente de la altitud, la temperatura y los procesos de producción primaria y descomposición de la materia orgánica. Asimismo, y como ya ha sido descrito por otros autores (Margalef, 1983), las mayores fluctuaciones de oxígeno disuelto se producen en tramos sometidos a una mayor contaminación y con una densidad importante de vegetación acuática. En este sentido, la concentración de oxígeno mostró una cierta variabilidad según los colectores analizados. Las menores concentraciones de oxí- 

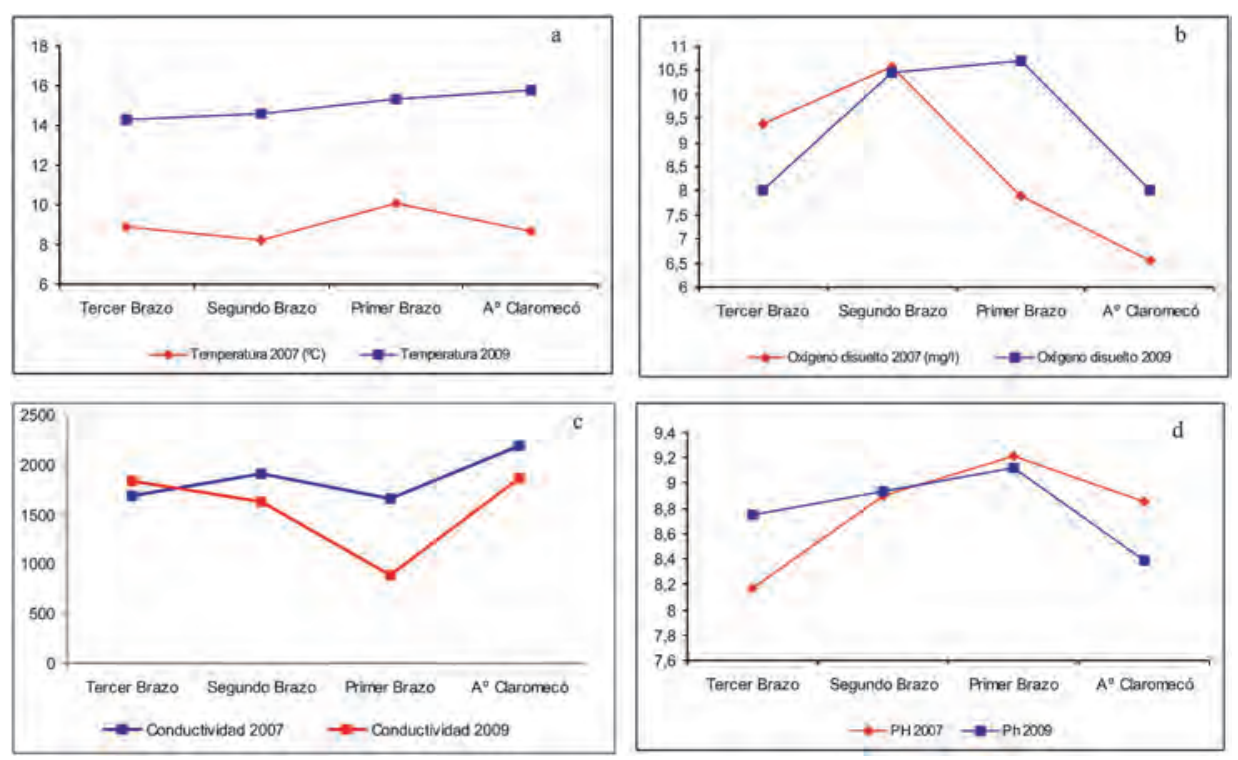

Figura 3. Variación de la temperatura (a), oxígeno disuelto (b), conductividad eléctrica (c) y pH (d) en la cuenca media del Arroyo Claromecó entre agosto de 2007 y agosto 2009.

geno disuelto se han encontrado en el tramo principal del Claromecó, con un total de $6.54 \mathrm{mg} \mathrm{l}^{-1} \mathrm{y}_{\text {de }} 8 \mathrm{mg} \mathrm{l}^{-1}$ para 2007 y 2009 respectivamente. Los valores máximos están en el Segundo Brazo, con $10.57 \mathrm{mg} \mathrm{l}^{-1}$ para 2007 si bien en 2009 el Primer Brazo registró una mayor concentración con valores de hasta $10.7 \mathrm{mg} \mathrm{l}^{-1}$ (Fig. 3b). En todas las estaciones medidas los valores son mayores a $5 \mathrm{mg} \mathrm{l}^{-1}$ de acuerdo a los límites tolerables para el desarrollo de la vida acuática.

Tabla 1. Valores mínimos y máximos de los parámetros físicos y químicos obtenidos durante el año 2007 y 2009 en la cuenca media del Arroyo Claromecó.

\begin{tabular}{|l|c|c|c|}
\hline Parámetro & & $\mathbf{2 0 0 7}$ & $\mathbf{2 0 0 9}$ \\
\hline Temperatura del agua & ${ }^{\circ} \mathrm{C}$ & $8.2-10.1$ & $14.3-15.8$ \\
\hline $\mathrm{pH}$ & & $8.1-9.21$ & $8.3-9.12$ \\
\hline Oxígeno disuelto & $\mathrm{mg} \mathrm{l}^{-1}$ & $6.5-10.5$ & $8-10.7$ \\
\hline Conductividad & $\mu \mathrm{S} \mathrm{cm}^{-1}$ & $1.66-2.19$ & $0.89-1.86$ \\
\hline Amonio & $\mu \mathrm{mol} \mathrm{l}^{-1}$ & $4.8-106$ & $3.04-150$ \\
\hline Nitratos & $\mu \mathrm{mol} \mathrm{l}^{-1}$ & $199-301$ & $143-287$ \\
\hline Nitritos & $\mu \mathrm{mol} \mathrm{l}^{-1}$ & $0.7-11.2$ & $2.15-30.1$ \\
\hline Fosfatos & $\mu \mathrm{mol} \mathrm{l}^{-1}$ & $6.2-109$ & $1.14-18.9$ \\
\hline Clorofila "a" & $\mathrm{mg} \mathrm{m}^{-3}$ & $1.4-8.2$ & $0.7-10.2$ \\
\hline MOP & $\mathrm{mgC} \mathrm{m}^{-3}$ & $522-1100$ & $317-820$ \\
\hline Feopigmentos & $\mathrm{mg} \mathrm{m}^{-3}$ & $3.2-15$ & $3.05-25$ \\
\hline
\end{tabular}



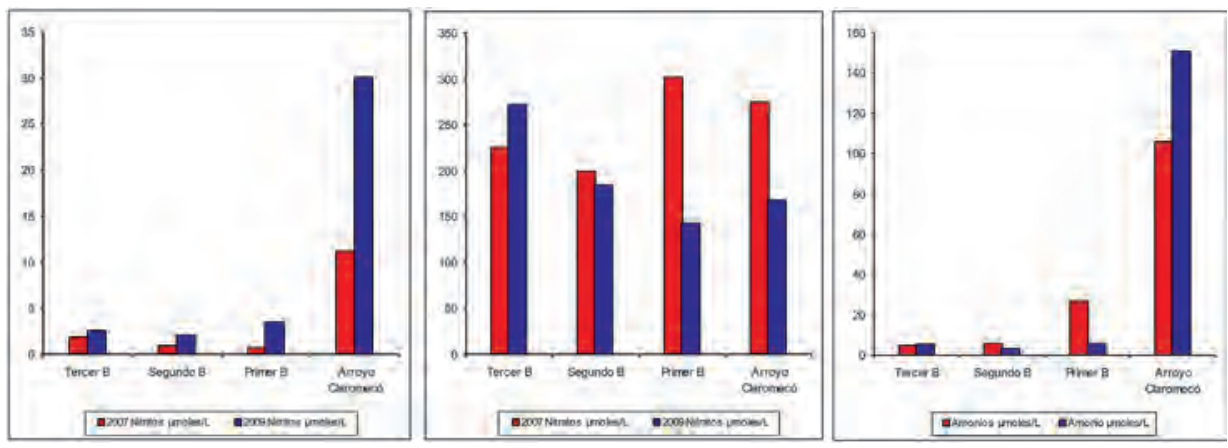

Figura 4. Concentración de nitritos, nitrato y amonio en los cursos de la cuenca media del Arroyo Claromecó en agosto de 2007 y 2009.

La conductividad presentó un máximo valor en la estación del Arroyo Claromecó durante el año 2007, alcanzando los $2190 \mu \mathrm{s} \mathrm{cm}^{-1}$. Los valores de este parámetro disminuyeron considerablemente para el Primer Brazo y para el Arroyo Claromecó durante el año 2009. Estos valores, en condiciones naturales, pueden deberse a la solubilidad del terreno, a la alta evaporación y a las escasas precipitaciones, propias de la zona en esa época del año y que no superaron los $4 \mathrm{~mm}$ mensuales durante los 2 períodos (Fig. 3c).

Los valores de nutrientes que se obtienen en el análisis de las muestras de agua en los tres brazos indican valores de nitrógeno-nitratos de escasa irregularidad entre los distintos colectores, y pueden marcar el predominio, en la cuenca, de materiales ricos en rocas evaporíticas, al menos en estos tres afluentes. Por el contrario, la disparidad existente en las concentraciones de nitritos y amonio entre éstos y el tramo principal del Claromecó, arroja la existencia de una cierta anomalía en este último (Fig. 4). En este sentido, la concentración de nitritos en el Arroyo Claromecó supera los $11 \mu \mathrm{mol} \mathrm{l}^{-1} \mathrm{y}$ $30 \mu \mathrm{mol} \mathrm{l}^{-1}$ para 2007 y 2009 respectivamente, un valor muy superior al del resto de colectores, que en ningún caso alcanza los $3.5 \mu \mathrm{mol} \mathrm{l}^{-1}$. Asimismo, la concentración de amonio llega a $150 \mathrm{umol} \mathrm{l}^{-1}$ en el Claromecó para 2009, mientras que en el resto oscila entre 3 y $26 \mu \mathrm{mol} \mathrm{l}^{-1}$ para 2007 y 2009 . Con respecto a los nitratos, los valores oscilaron entre 175 y $301 \mu \mathrm{mol} \mathrm{l}^{-1}$ para todos los colectores medidos, y se observó una tendencia a la disminución de la concentración de los mismos para el año 2009, principalmente en el Primer Brazo y en el Arroyo Claromecó.

Si se consideran las especies químicas del fósforo, los resultados del análisis de la concentración de fosfatos (Fig. 5) indican que los niveles también fluctuaron bastante entre los tres afluentes, en los que la presencia de fosfatos mantiene unos valores próximos, y el tramo principal del Claromecó, en el que los niveles alcanzaron los $109.80 \mu \mathrm{mol} \mathrm{l}^{-1}$ para el año 2007. Los valores de fosfatos para la medición de 2009 disminuyeron notablemente en los tres brazos, aunque el tramo principal también presenta el mayor valor con $18.9 \mu \mathrm{mol} \mathrm{l}^{-1}$, muy inferiores al año 2007. 
Figura 5. Concentración de fosfatos y silicatos en los cursos de la cuenca media del Arroyo Claromecó en agosto de 2007 y 2009.
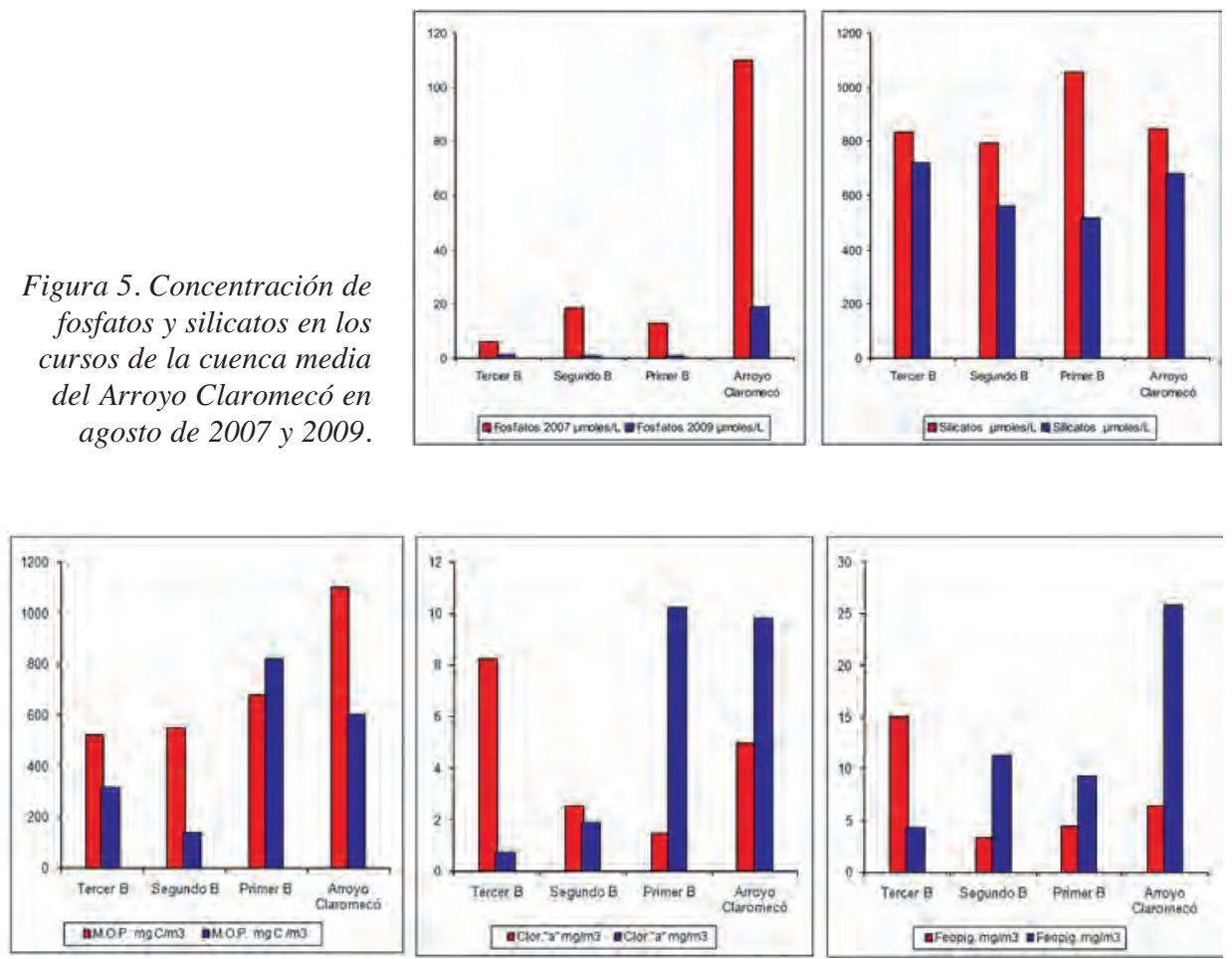

Figura 6. Concentración de MOP, clorofila "a" y feopigmentos en la Cuenca del Arroyo Claromecó en agosto de 2007 y 2009.

La concentración de silicatos durante ambas mediciones superó los $500 \mu \mathrm{mol} \mathrm{^{-1 }}$. En la medición del 2007 los valores fueron superiores en todos los colectores, el máximo se presentó en el Primer Brazo con $1054 \mu \mathrm{mol} \mathrm{1} 1^{-1}$. Durante la segunda medición los valores disminuyeron considerablemente en el Primer Brazo.

Los valores de MOP presentaron para el año 2007 un máximo en el curso principal de $1100 \mathrm{mgC} \mathrm{m}^{-3}$ mientras que en el año 2009 el máximo correspondió al Tercer Brazo con un valor de $819 \mathrm{mgC} \mathrm{m}^{-3}$ (Fig. 6). En cuanto a los pigmentos, el valor de clorofila "a" fue máximo en el Primer Brazo con un total de $10.22 \mathrm{mg} \mathrm{m}^{-3}$. La mayoría de las observaciones variaron entre 0.7 y $9.8 \mathrm{mg} \mathrm{m}^{-3}$ para los años 2007 y 2009 . Por su parte, los feopigmentos alcanzaron su valor máximo en el curso principal, con $25.7 \mathrm{mg} \mathrm{m}^{-3}$, mientras que en el resto de colectores los valores oscilaron entre 3 y $15 \mathrm{mg} \mathrm{m}^{-3}$ en forma comparada entre agosto 2007 y agosto 2009.

En la Fig. 7 se presentan las concentraciones de los metales pesados analizados en las aguas superficiales de cada uno de los colectores. Los metales pesados analizados en los sitios de muestreo presentaron una significativa disminución entre el 2007 y 2009. Los valores de níquel (Ni) variaron entre 8.4 y $14.6 \mu \mathrm{g} \mathrm{g}^{-1}$ para la pri- 


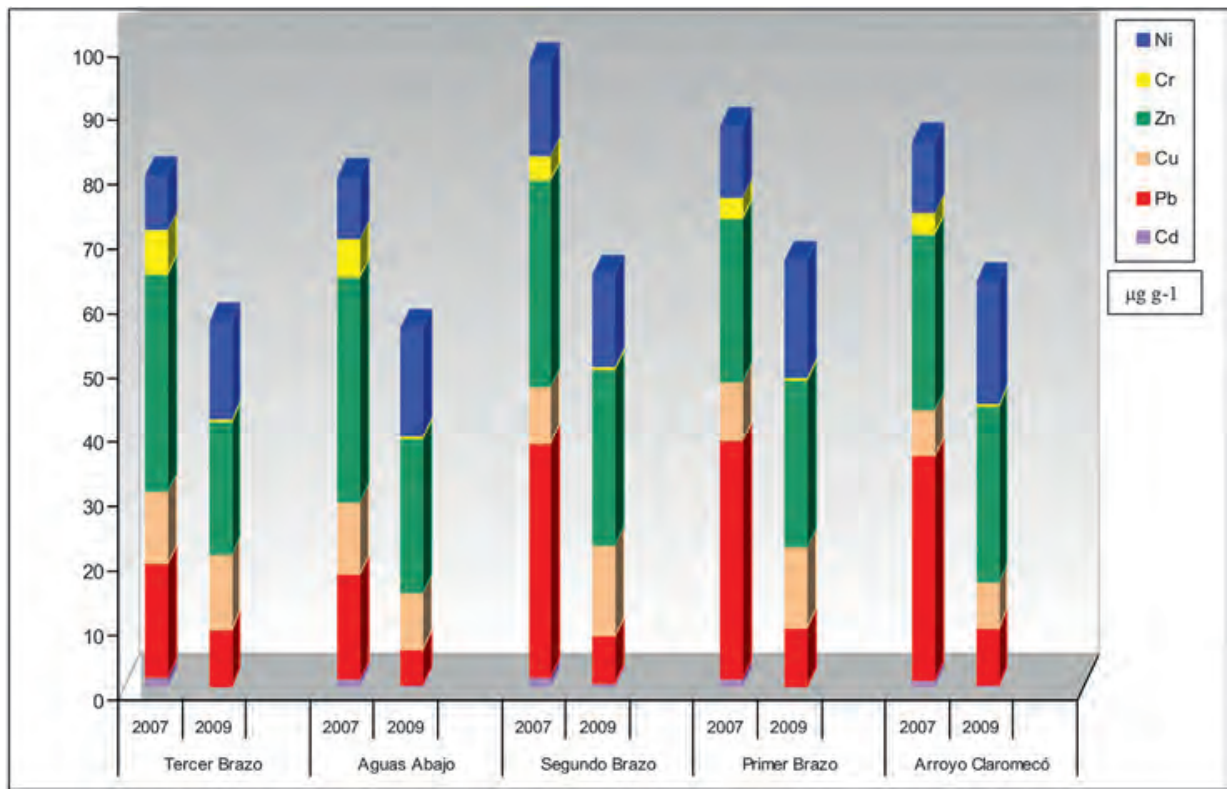

Figura 7. Valores de metales pesados hallados en los sedimentos fluviales en la cuenca del Arroyo Claromecó (2007-2009).

mera medición mientras que en la segunda medición los valores oscilaron entre 14.4 y $18.9 \mu \mathrm{g} \mathrm{g}^{-1}$. El cromo $(\mathrm{Cr})$ tiene mayor presencia en 2007 con valores entre $3.3 \mathrm{y}$ casi $7 \mu \mathrm{g} \mathrm{g}^{-1}$ en todos los colectores analizados, mientras que en el segundo muestreo es muy escasa su presencia $\left(0.4 \mu \mathrm{g} \mathrm{g}^{-1}\right)$. El zinc $(\mathrm{Zn})$ varió entre sendas mediciones: mientras que en el primer año el máximo llegó a $35 \mu \mathrm{g} \mathrm{g}^{-1}$, en el segundo alcanzó un total de $27 \mu \mathrm{g} \mathrm{g}^{-1}$. Los valores de cobre $(\mathrm{Cu})$ no presentaron variaciones entre ambas mediciones, oscilando entre 6.9 y $14.2 \mu \mathrm{g} \mathrm{g}^{-1}$ para el curso principal y el Segundo Brazo respectivamente. Se detectó presencia de plomo $(\mathrm{Pb})$ en los diferentes sitios de muestreo en el año 2007, con valores máximos de hasta $37 \mu \mathrm{g} \mathrm{g}^{-1}$ para el Primer Brazo y un mínimo de $16 \mu \mathrm{g} \mathrm{g}^{-1}$ para el Tercer Brazo en sus dos mediciones. En agosto de 2009 los valores disminuyeron considerablemente $\left(5.5 \mathrm{y} \mu \mathrm{g} \mathrm{g}^{-1}\right)$. Los valores de cadmio (Cd) oscilaron entre 1.3 y $0.22 \mu \mathrm{g} \mathrm{g}^{-1}$ para ambos muestreos, los mínimos se observaron durante la ultima medición. La variación de plomo y cromo es la más llamativa para cada uno de los cursos estudiados siendo el Tercer Brazo y Claromecó los que poseen la mayor diferencia. El Tercer Brazo fue analizado en dos sitios de muestreo y también sigue una tendencia de las concentraciones a la baja.

\section{Discusión}

Las aguas de los arroyos de este sector de la cuenca del Claromecó son naturalmente alcalinas debido principalmente al tipo de sustrato calcáreo que caracteriza a esta zona de la llanura pampeana. Los valores de la temperatura del agua hallados son nor- 
males para cursos de agua de la zona del sudeste bonaerense (Marini et al. 2002; Herzer et al. 2003). Los valores de la conductividad no superaron los niveles establecidos para la protección de la vida acuática que indica como límite $2500 \mu \mathrm{s} \mathrm{cm}^{-1}$. Aunque se debe aclarar que los aumentos puntuales de este parámetro se presentaron en el curso principal Claromecó, asociado probablemente al vertido de aguas residuales al sistema fluvial como también lo señala Peluso et al. (2011), referido al riesgo sanitario que poseen estos cursos fluviales para uso recreativo.

En este sentido, y en base a las condiciones físico-químicas del agua, se podría decir que, el Primer, Segundo y Tercer Brazo reflejan las características propias del sustrato de la cuenca del Arroyo Claromecó. Mientras que, por el contrario, el arroyo Claromecó presenta valores muy dispares de amonio, fosfatos y oxígeno disuelto, asociado a posibles descargas domésticas al río principal, en su tramo bajo. Los valores de nutrientes que se obtienen en el análisis de las muestras de agua de los distintos colectores del Claromecó se consideran intermedios para sistemas acuáticos en el Primer, Segundo y Tercer Brazo de la red del Claromecó. Los valores que presenta el curso principal indican un nivel intermedio respecto a los niveles guía para la protección de la vida acuática. No se han podido hacer estudios estadísticos dado que estos cursos han sido recientemente monitorizados y no existen antecedentes de datos históricos; solamente se han evaluado otros sectores de la cuenca del Claromecó para riego complementario.

Las concentraciones de fosfatos y nitrógeno son mayores en el Arroyo Claromecó, tendencia que se mantiene con casi todos los parámetros químicos, lo que se correspondería a descargas cloacales y secundariamente industriales de las plantas procesadoras por las que atraviesa este curso fluvial en el área urbana periurbana de la localidad de Tres Arroyos. Esta condición también se indica en el trabajo de Peluso et al. (2011). El enriquecimiento de las aguas con fosfatos se debe a los efluentes domésticos, detergentes, jabones en polvo y pesticidas (Varni et al., 2006), tal y como se observa en el tramo principal (Fig. 2d). Los valores de MOP en los tres cursos no superaron los niveles aceptables para la vida acuática, que han de ser de hasta $700 \mathrm{mgC} \mathrm{m}^{-3}$. No obstante, sí fue superior en el tramo principal del Claromecó. Los niveles de los pigmentos tampoco superaron los niveles óptimos para la vida acuática, que han de estar entre 100 y $150 \mathrm{mg} \mathrm{m}^{-2}$, si bien el máximo valor lo presentó el Tercer Brazo durante el año 2009 con $102 \mathrm{mg} \mathrm{m}^{-2}$. Los valores de feopigmentos no superaron los $26 \mathrm{mg} \mathrm{m}^{-3}$ en concordancia con los niveles aceptados para la protección de la vida acuática.

La concentración de nitratos para los períodos muestreados presenta valores superiores al límite establecido como nivel guía para la protección de la vida acuática en cursos superficiales dulces, que es de $50 \mathrm{mg} \mathrm{l}^{-1}$. Esto concuerda con numerosos estudios realizados en aguas superficiales de la provincia de Buenos Aires en este sector de la llanura pampeana, que han demostrado la relación positiva que existe entre el porcentaje de uso de suelo agrícola en el sector y las concentraciones de nitratos en las aguas de los arroyos (Sala et al., 1983; William et al., 2005; Dodds y Oakes, 2006; Varni et al., 2006; Peluso et al., 2011). Amuchástegui (2006) realizó un análisis de concentración de nitratos en 23 arroyos de la provincia de Buenos Aires, donde el uso de suelo agrícola mixto está altamente relacionado con la alta concentración de ese parámetro, que varía entre 
34 y $3450 \mu \mathrm{mol} \mathrm{l}^{-1}$. Los excesos de nitrógeno provenientes de la actividad agrícola llegan rápidamente al caudal de los arroyos pampeanos (Sala et al., 1983). Asimismo, varios autores consideran el estado natural de la vegetación de ribera como una medida de mitigación en la contaminación por agroquímicos dado que la misma favorece la denitrificación (Pizarro et al., 2005; Amuchástegui, 2006; Feijoó y Lombardo, 2007). Las riberas de los cursos de agua estudiados se hallan en parte modificadas pues se observan usos agrícolas y ganaderos en las mismas, además de vegetación introducida en las riberas del curso principal (García Martínez et al., 2008).

Los valores de metales pesados encontrados en los distintos colectores indican concentraciones bajas de cadmio, cobre, zinc y cromo, concentraciones normales de níquel y manganeso y concentraciones intermedias de plomo. La variación más significativa de la concentración la presentan el plomo y el cobre entre el muestreo de 2007 y 2009. Esta condición se podría explicar a partir de la intensificación de la agricultura en los campos aledaños. La incorporación de las nuevas técnicas de siembra (entre ellas la siembra directa que demanda el continuo uso de fertilizantes y pesticidas) podría explicar el valor intermedio de plomo en los colectores de la red para el año 2007. La variabilidad marcada en esas concentraciones tendría una leve explicación en la disminución del uso de herbicidas, principalmente ocurridos en el 2009, dado que las condiciones de sequía en los campos retrasó la preparación de los suelos para cultivos en cuanto a la aplicación de agroquímicos se refiere.

\section{Conclusiones}

La calidad del agua y de los sedimentos de los cursos de la cuenca media del Arroyo Claromecó depende de diversos factores ambientales como el sustrato geológico, la topografía de los terrenos donde se ubican estos cursos y principalmente los usos del suelo. En cuanto a los parámetros físicos obtenidos, los tres brazos del Arroyo Claromecó evidenciaron valores similares para cada año de medición, mientras que el curso principal presentó mayor variación.

Los valores de nutrientes hallados presentaron mayor variabilidad entre los años 2007 y 2009. Los máximos se observaron durante el primer año de medición, mientras que el máximo de amonio se observó en el colector principal para el año 2009. Se detectó una presencia de nitratos superior a los niveles guía de protección de la vida acuática de acuerdo a los valores encontrados en arroyos de la provincia de Buenos Aires. Las concentraciones de fosfatos y nitrógeno fueron mayores en el Arroyo Claromecó, tendencia que se mantuvo con casi todos los parámetros químicos, lo que se correspondería a descargas cloacales y secundariamente industriales de las plantas procesadoras por las que atraviesa este curso fluvial en el área urbana periurbana de la localidad de Tres Arroyos.

Los sedimentos fluviales analizados presentaron valores de metales pesados intermedios. Los primeros corresponden a $\mathrm{Cd}, \mathrm{Cr}, \mathrm{Zn}$ y $\mathrm{Cu}$. Los valores normales corresponden a $\mathrm{Ni}, \mathrm{Mn}$ y $\mathrm{Fe}$. La influencia de las actividades agrícolas y ganaderas en el entorno de los cursos fluviales analizados puede observarse incipientemente en el análisis de los datos de la concentración de plomo de todos los colectores para el año 2007 que resultaron valores intermedios. 
El presente estudio permitió advertir que los cursos superiores de la cuenca media del Arroyo Claromecó se hallan relacionados con las características hidrogeológicas y usos agrícolas y ganaderos, y el curso principal se halla influenciado principalmente por el uso urbano e industrial. La calidad físico-química del agua de este sistema fluvial no representa un riesgo para la vida acuática en general, ni tampoco si se usa para riego suplementario o bebida para animales. La monitorización continua de estos sistemas fluviales permitirá poseer herramientas eficientes de manejo y gestión de los mismos.

\section{Referencias bibliográficas}

Amuchástegui, G. 2006. Relationships among water chemistry, fisiographic features and land use in Pampean streams. Tesis de Grado. National University of Lujan, Lujan, 70 pp.

Andrade Couce, M.L., Fernández Covelo, E., Alonso Vega, M.F. 2005. Influencia del manejo agrícola intensivo en la contaminación del suelo. Revista Pilquen - Sección Agronomía 7, 1-17.

Carbone, M.E. 2004. Hidrografía del Arroyo Claromecó. Tesis Doctoral. Departamento de Geografía, Universidad Nacional del Sur, Bahía Blanca, 195 pp.

Carbone, M.E., Píccolo, M.C., Perillo, G.M.E. 2005. Evaluation of the surface waters of the Claromecó River Basin for supplementary irrigation. Revista Geográfica 137.

Carbone, M.E., Píccolo, M.C., Perillo, G.M.E. 2006. Comportamiento hidrológico del arroyo Claromecó, Argentina. Investigaciones Geográficas 39, 179-193.

CEI Barrow 2003. Estimaciones de área sembrada-Campaña 2002/03. Chacra Experimental Integrada Barrow I.N.T.A., Tres Arroyos, Buenos Aires, pp. 1-5.

Dodds, W.K., Oakes, R.M. 2006. Controls of nutrients across a prairie stream watershed: landuse and riparian cover effects. Environmental Management 37, 634-646.

Eberlein, K., Kattner, G. 1987. Automatic method for the determination of orthophosphate and total dissolved phosphorous in the marine environment. Fresenius' Zeitschrift für Analytische Chemie 326, 354-357.

Feijoo C.S., Lombardo R J. 2007. Baseline water quality and macrophyte assemblages in Pampean streams: A regional approach. Water Research 41, 1399-1410.

Forján, H. 2004. Rotaciones en sistemas mixtos. Informe Técnico. Chacra Experimental Integrada Barrow I.N.T.A., Tres Arroyos, Buenos Aires, pp. 9-18.

García Martínez, B., Carbone, M.E., Piccolo, M.C., Perillo, G.M.E. 2008. Incidencia de la variabilidad hidrológica en la morfología de cauces del arroyo Claromecó (Buenos Aires, Argentina). Geographicalia 54, 143-160.

Goic, L., Rojas, C. 2004. Sistemas de crianza, de recría y engorda en la zona sur. En Manual de producción de bovinos de carne para la VIII, IX y X regiones, C.M. Rojas, A. Doussoulin, A. Olivares (eds.). Instituto de Investigaciones Agropecuarias, Centro Regional de Investigación Carillanca y Fundación para la Innovación Agraria, Temuco, pp. 107-120.

Grasshoff, K., Erhardt, M., Kremling, K. 1983. Methods of Seawater Analysis. Verlag Chemie, Weinheim, New York, 317 pp.

Herzer, H., Celis, A., Bartolomé, M., Rodríguez, C., Caputo, G. 2003. El manejo de cuencas y su impacto en áreas urbana: el caso de la llanura pampeana, Argentina. III Congreso Latinoamericano de manejo de cuencas hidrográficas. INRENA-FAO, Arequipa, 12 pp. Disponible en: http://www.cesam.org.ar.

Instituto Nacional de Estadística y Censos (INDEC) 2010. Censo Nacional de Población, Hogares y Viviendas. Disponible en: http://www.indec.gov.ar. 
Lerda, D., Prosperi, C. 1994. Consideraciones sobre la potabilización del agua para consumo humano en Río Tercero (Córdoba, Argentina). Ayuntamiento de Marcos Juárez, pp. 1-42.

Mancini, M., Rodríguez, C., Prosperi, C., Finola, M. 2003. Monitoreo de reservorios del centro de Córdoba (Argentina) como base para una adecuada gestión ambiental. En Actas del Congreso Argentino de Saneamiento y Medio Ambiente, AIDIS, Buenos Aires, pp.1-19.

Marcovecchio, J.E., Moreno, V.J., Pérez, A. 1988. Determination of heavy metal concentrations in biota of Bahía Blanca, Argentina. The Science Total Environment 75, 181-190.

Marini, M.F. 2002. Hidrografia del río Quequén Salado. Tesis Doctoral. Universidad Nacional del Sur, Bahía Blanca, 162 pp.

Margalef, R. 1983. Limnología. Omega, Barcelona.

Márquez, A., Martínez, G. 2000. Concentración y comportamiento de metales pesados en una zona estuarina de Venezuela. Interciencia 25, 284-291.

Mason J.P., Miller, K. 2004. Water Resources of Sweetwater Country, Wyoming. Scientific Investigations Report 2004-5214, pp 21-44.

Mencio, A., Maspla, J. 2008. Assessment by multivariate analysis of ground water surface water interactions in urbanized Mediterranean streams. Journal of Hydrology 352, 355-366.

Panno, S.V., Kelly, W.R., Hackley, K.C., Hwang, H.H., Martinsek, A.T. 2008. Sources and fate of nitrate in the Illinois River Basin, Illinois. Journal of Hydrology 359, 174-188.

Peluso, F., González Castelain, J., Othax, N., Rodríguez, L. 2011. Riesgo sanitario por sustancias tóxicas superficiales de Tres Arroyos, Argentina. Acta Bioquímica Clínica Latinoamericana 45 (2), 311-321.

Perona, E., Bonilla, I., Mateo, P. 1999. Spatial and temporal changes in water quality in a Spanish river. The Science of the Total Environment 241, 75-90.

Pizarro, H., Alemanni, M.E. 2005. Variables físico-químicas del agua y su influencia en la biomasa del perifiton en un tramo inferior del Río Luján (Provincia de Buenos Aires). Ecología Austral 15, 73-88.

Portela, S., Andriulo, A., Sasal, M.C., Mary, B., Jobbigy, E. 2006. Fertilizer vs. organic matter contribution to nitrogen leaching in cropping systems of the pampas: $15 \mathrm{~N}$ applications in field lysimiters. Plant Soil 289, 265-277.

Sala, J.M., González, N., Kruse, E. 1983. Generalización hidrológica de la Provincia de Buenos Aires. En Hidrología de las grandes llanuras: Actas del Coloquio de Olavarria, UNESCO - Ministerio de Obras y Servicios Públicos, Buenos Aires, pp. 974-1009.

Williams, M., Hopkinson, C., Rastetter, E., Vallino, J., Claessens, L. 2005. Relationships of land use and stream solute concentrations in the Ipswich River basin, Northeastern Massachusetts. Water, Air and Soil Pollution 161, 55-74.

Zamora, M., Melin, A.A., Andrés, E. 2009. Sorgo. Graníferos RED SUR 2008/09, Informe técnico. 